\title{
Hubble Space Telescope Snapshot Search for Planetary Nebulae in Globular Clusters of the Local Group ${ }^{1}$
}

\author{
Howard E. Bond ${ }^{2,3}$
}

Received —

\footnotetext{
${ }^{1}$ Based on observations with the NASA/ESA Hubble Space Telescope obtained at the Space Telescope Science Institute, and from the data archive at STScI, which are operated by the Association of Universities for Research in Astronomy, Inc., under NASA contract NAS5-26555

${ }^{2}$ Department of Astronomy \& Astrophysics, Pennsylvania State University, University Park, PA 16802, USA; heb11@psu.edu

${ }^{3}$ Space Telescope Science Institute, 3700 San Martin Dr., Baltimore, MD 21218, USA
} 


\begin{abstract}
Single stars in ancient globular clusters (GCs) are believed incapable of producing planetary nebulae (PNe), because their post-asymptotic-giant-branch evolutionary timescales are slower than the dissipation timescales for PNe. Nevertheless, four PNe are known in Galactic GCs. Their existence likely requires more exotic evolutionary channels, including stellar mergers and common-envelope binary interactions. I carried out a snapshot imaging search with the Hubble Space Telescope $(H S T)$ for PNe in bright Local Group GCs outside the Milky Way. I used a filter covering the $5007 \AA$ nebular emission line of [O III], and another one in the nearby continuum, to image $66 \mathrm{GCs}$. Inclusion of archival $H S T$ frames brought the total number of extragalactic GCs imaged at $5007 \AA$ to 75 , whose total luminosity slightly exceeds that of the entire Galactic GC system. I found no convincing PNe in these clusters, aside from one PN in a young M31 cluster misclassified as a GC, and two PNe at such large angular separations from an M31 GC that membership is doubtful. In a ground-based spectroscopic survey of 274 old GCs in M31, Jacoby et al. (2013) found three candidate PNe. My HST images of one of them suggest that the [O III] emission actually arises from ambient interstellar medium rather than a $\mathrm{PN}$; for the other two candidates, there are broad-band archival UV HST images that show bright, blue point sources that are probably the PNe. In a literature search, I also identified five further PN candidates lying near old GCs in M31, for which follow-up observations are necessary to confirm their membership. The rates of incidence of PNe are similar, and small but non-zero, throughout the GCs of the Local Group.
\end{abstract}

Subject headings: globular clusters: general — planetary nebulae: general — stars: AGB and post-AGB — stars: evolution — stars: mass loss 


\section{Introduction: Planetary Nebulae in Globular Clusters}

The first planetary nebula (PN) belonging to a globular cluster (GC) was discovered more than 85 years ago, in M15 (Pease 1928). It was another six decades before a second GCPN was found, this time in M22 (Gillett et al. 1989). Jacoby et al. (1997, hereafter JMF97) then carried out a systematic ground-based CCD survey of 133 Milky Way GCs, using a narrow-band [O III] $5007 \AA$ filter along with a filter in the neighboring continuum. They discovered two more PNe, in the Galactic clusters NGC 6441 and Pal 6. The number of PNe known in GCs in the Local Group was raised to five by the serendipitous discovery of a PN in the cluster H5 belonging to the Fornax dwarf spheroidal galaxy (Larsen 2008). Outside the Local Group, [O III] emission has been detected in the integrated light of a handful of GCs during spectroscopic investigations, as summarized by Minniti \& Rejkuba (2002), Zepf et al. (2008), Chomiuk, Strader, \& Brodie (2008), and Peacock, Zepf, \& Maccarone (2012). (However, as discussed in $§ 5$, not all of these distant emission sources are actually PNe.)

PNe in GCs raise two issues related to stellar evolution. The first is Why are there so few PNe in GCs? JMF97 posed this question because one would expect to find $\sim 16$ PNe in the Milky Way GCs on the basis of the total luminosity of the Galactic GC system, a PN lifetime of $\sim 2-3 \times 10^{4} \mathrm{yr}$, and the assumption that every star produces a visible PN near the end of its life. (The prediction comes basically from an application of the "fuel-consumption theorem," as defined by Renzini \& Buzzoni 1986.) In order to explain the smaller number actually observed, JMF97 suggested that the assumption that every star produces a PN may be incorrect in GCs. In fact, single stars in very old populations, having started their lives at about $0.80 M_{\odot}$, leave the asymptotic giant branch (AGB) with masses reduced to as low as $\sim 0.50 M_{\odot}$ (Alves, Bond, \& Livio 2000; hereafter ABL00) to $\sim 0.53 M_{\odot}$ (Kalirai et

al. 2009). The theoretical post-AGB evolutionary timescales of such low-mass remnants are 
so long (e.g., Schoenberner 1983) that any nebular material ejected at the end of the AGB phase has time to disperse before the central star becomes hot enough to ionize it. Thus, the single stars now evolving in GCs would not be expected to produce any visible ionized PNe.

Now the question becomes Why are there any PNe in GCs at all? The answer probably lies in the evolution of binary stars. There are (at least) two ways that binaries can produce PNe in populations in which single stars cannot. (1) Coalescence of two stars in a binary near the main sequence could produce first a blue straggler, and eventually a higher-mass post-AGB remnant that would evolve rapidly enough to ionize a PN. ABL00 detected no photometric variations for K648, the central star of the PN Ps 1 in M15, consistent with it being a merger remnant. (2) Or a red giant or AGB star may undergo a common-envelope $(\mathrm{CE})$ interaction with a companion, rapidly exposing the giant's hot core, and thus promptly subjecting the ejecta to ionizing radiation. These and other scenarios to account for the presence of PNe in GCs have been discussed by ABL00, Ciardullo et al. (2005), Buell (2012), Jacoby et al. (2013; hereafter JCD13), and others. They are part of a larger conceptual framework in which it has been increasingly recognized that binary interactions are likely to be a major, if not dominant, formation channel for PNe in all populations (e.g., Bond \& Livio 1990; Bond 2000; De Marco 2009; and references therein).

The binary-merger hypothesis can be tested by determining the luminosities of central stars of PNe in GCs, and then inferring their masses from theoretical core-mass/luminosity relations. ABL00 used the Wide Field Planetary Camera 2 (WFPC2) on the Hubble Space Telescope (HST) to carry out photometry of K 648. The absolute luminosity of the star implied a mass of $0.60 M_{\odot}$. This is significantly higher than the masses of remnants of single stars in GCs (see above), giving the star a fast enough post-AGB evolution for it to have ionized the ejecta before they had time to dissipate. ABL00 concluded that the central star 
must have achieved its high mass as a result of a merger.

HST imaging of all four PNe in Galactic GCs, and photometry of their central stars, have been collected and discussed by Jacoby et al. (2014, 2015). Apart from K 648, the evidence for high stellar masses resulting from binary mergers has remained less compelling. In fact, if the PN were ejected as a consequence of a $\mathrm{CE}$ interaction, the mass of its central star would be unlikely to differ much from those of remnants of single-star evolution, or could even be lower. Jacoby et al. do, however, argue that the morphologies of these GCPNe are at least suggestive of ejection from a binary interaction. A potential test is to search for X-ray emission, arising from a synchronously rotating, active, late-type companion star to the PN nucleus. Variable X-ray emission from K 648 has in fact been detected by Hannikainen et al. (2005) — which, if due to the central star rather than the surrounding PN, would argue against the merger scenario I discussed above.

Further progress could be made with a larger sample than the five known PNe in the Milky Way and Fornax GC populations. There are many hundreds of GCs known in the Andromeda Galaxy, M31; and smaller numbers are known in M33, the Magellanic Clouds, and other members of the Local Group. As noted by many authors, galaxies like M31 have experienced different evolutionary histories than our Galaxy, which might be reflected in systematic differences in their GC systems (e.g., van den Bergh 2010 and references therein). Conceivably, for example, there could be a population of GCs with intermediate rather than extremely old ages, which would be richer in PNe than the Milky Way GCs.

JCD13 obtained integrated spectra of 274 old GCs in M31, searching for emission at [O III $5007 \AA$ that would indicate the presence of a PN in the cluster. Three candidate PNe were found among this sample of old GCs. In the complementary survey discussed here, I have used HST to search for PNe in GCs belonging to Local Group galaxies, especially M31, by obtaining direct narrow-band [O III] images. 


\section{Observations}

\subsection{HST Snapshot Scheduling}

In my search for PNe in Local Group GCs, I used HST in its "snapshot" mode, in which short exposures are inserted into gaps in the telescope schedule that remain once the primary observations have been sequenced as optimally as possible. My observations were made during two HST proposal cycles. In Cycle 16 (program ID SNAP-11218) I submitted a list of 125 target GCs, of which 41 were successfully observed. In Cycle 17 (SNAP-11714) I submitted 100 targets, of which 23 were observed. The Cycle 16 observations were made with WFPC2, from 2007 July 10 to 2008 November 23. In Cycle 17, I used the UVIS channel of the newly installed Wide Field Camera 3 (WFC3), and these observations were made between 2009 September 1 and 2011 September 29. Nine of the Cycle 17 observations were repeats of WFPC2 targets, using the more sensitive WFC3. Eleven additional GCs were serendipitously present in the frames along with the primary targets, for a total of 66 unique clusters, belonging to eight different galaxies.

\subsection{Target Selection}

The list of candidate targets for both HST Cycles was developed as follows. The overall aim was primarily to maximize the total integrated luminosity of the cluster sample, in order to maximize the probability of discovering $\mathrm{PNe}$; and secondarily to provide targets widely distributed across the sky. (1) For the Magellanic Clouds, I chose the 8 "Population II" clusters with integrated visual absolute magnitudes of $M_{V} \leq-7.5$, as listed by Olszewski, Suntzeff, \& Mateo (1996, their Table 1). (2) In M33, I selected the 5 brightest red (i.e., old) GCs listed by Christian \& Schommer (1988, their Table VII); all of them are brighter than $V=16.5\left(M_{V} \leq-8.0\right)$. (3) In the dwarf irregular galaxies NGC 6822 
and WLM, I chose Hubble VII in the former, and WLM-1 in the latter. Hubble VII, with an integrated $V=15.8\left(M_{V}=-7.7\right)$ is an old, metal-poor GC (e.g., Wyder, Hodge, \& Zucker 2000). WLM-1 is the lone GC known in its galaxy, and is also very old (e.g., Hodge et al. 1999, who give $V=16.1$ and $M_{V}=-8.8$ ). (4) For the Fornax dwarf spheroidal, out of its five known GCs I included the two bright clusters NGC 1049 and H2, for which Strader et al. (2003) give $V$ magnitudes of 12.6 and $13.5\left(M_{V}=-8.1\right.$ and -7.2$)$. (5) For the dwarf elliptical NGC 147, I selected Hodge II, with a $V$ magnitude of 16.5 (Sharina et al. 2006), corresponding to $M_{V}=-7.4$. (6) I then selected GCs in the M31 system in order of increasing integrated $V$ magnitudes from the catalog of Galleti et al. (2004), which is maintained online 1$]$ until the target lists were filled to the allocated numbers. These lists contained clusters with integrated $V$ magnitudes ranging from 13.8 to $16.8\left(M_{V}=-10.6\right.$ to $-7.6)$

\subsection{Observing Strategy}

With both WFPC2 and WFC3, I observed each cluster in the narrow-band F502N [O III] $5007 \AA$ filter, and the broad-band F555W " $V$ " filter. Exposure times for most of the GCs were $2 \times 500 \mathrm{~s}$ and $2 \times 100 \mathrm{~s}$ in F502N and F555W, respectively, for WFPC2, or $2 \times 300 \mathrm{~s}$ and $2 \times 60 \mathrm{~s}$ in F502N and F555W for WFC3. For the nearby Magellanic Cloud GCs, the WFPC2 exposures were shortened to $2 \times 200 \mathrm{~s}$ and $2 \times 30 \mathrm{~s}$.

The field of view of WFPC2 is $35^{\prime \prime} \times 35^{\prime \prime}$ for the PC chip (I placed the target GCs near the corner of the PC field least affected by charge-transfer inefficiency [CTI]), plus $76^{\prime \prime} \times 76^{\prime \prime}$ for each of the three neighboring wide-field (WF) chips. The plate scale is $0{ }^{\prime \prime} 046$ pixel $^{-1}$

\footnotetext{
${ }^{1}$ At http://www.bo.astro.it/M31. Another useful catalog of M31 GCs is Peacock et
} al. (2010). 
for PC, and $0{ }^{\prime \prime} 100$ pixel $^{-1}$ for the WF. For the WFC3 imaging I centered each target in a $1024 \times 1024$ pixel subarray, and the plate scale is $0^{\prime \prime} 039$ pixel $^{-1}$, giving a field of view of $40^{\prime \prime} \times 40^{\prime \prime}$.

Table 1 lists the 66 GCs that were imaged. The nomenclature is as given in $\S 2.2$; for M31 I have used the designations of the Galleti et al. (2004) and Peacock et al. (2010) catalogs. Column 2 gives their integrated visual absolute magnitudes, calculated using integrated $V$ magnitudes and distances and reddenings from the sources cited in the previous subsection. Serendipitously, nine of the WFPC2 frames contained a second, usually fainter GC in one of the WF chips, as indicated in the footnotes to the table. In one case, the smaller WFC3 field contained a second cluster: NGC 147 Hodge III ( $V=17.0$, $\left.M_{V}=-6.9\right)$ was in the field of view when Hodge II was observed.

All exposures were dithered for cosmic-ray removal by taking two exposures in each filter and moving the telescope pointing by a few pixels between exposures. For the WFPC2 frames, I used IRAF2 routines to align and combine them, while for WFC3 I used the pipeline drizzle-combined images from the $H S T$ archive.

\section{Searching for $\mathrm{PNe}$}

\subsection{Search Techniques}

A PN will be bright in the narrow-band F502N filter centered near the emission line of [O III] at $5007 \AA$. The broad-band F555W filter also transmits at $5007 \AA$ (where the system

\footnotetext{
${ }^{2}$ IRAF is distributed by the National Optical Astronomy Observatories, which are operated by the Association of Universities for Research in Astronomy, Inc., under cooperative agreement with the National Science Foundation.
} 
throughput is actually higher than for F502N), as well as at other emission lines in PNe, the strongest of which are [O III] $4959 \AA$, $\mathrm{H} \alpha$ and $\mathrm{H} \beta$, and He I $5876 \AA$. To estimate the expected relative count rates for a typical PN in these two filters, I used the line intensities for the halo PN BoBn 1 given by Otsuka et al. (2010). I convolved them with the system throughputs for both cameras in the two filters, as given in the respective Instrument Handbooks (WFPC2: McMaster \& Biretta 2008; WFC3: Bond \& Quijano 2007). The resulting predicted count-rate ratios for the $\mathrm{PN}$ are $\mathrm{F} 555 \mathrm{~W} / \mathrm{F} 502 \mathrm{~N}=2.5$ for $\mathrm{WFPC} 2$, and 2.1 for $\mathrm{WFC} 3$.

Drizzled WFC3 images from the $H S T$ archive are in units of counts $\mathrm{s}^{-1}$, so a typical PN would be approximately twice as bright in F555W as in F502N. However, WFPC2 images from the archive pipeline give total counts accumulated during the exposure. Since the ratio of exposure times was $\mathrm{F} 502 \mathrm{~N} / \mathrm{F} 555 \mathrm{~W}=5$ (in most cases) for WFPC2, the ratio of expected integrated counts for a $\mathrm{PN}$ in the two filters is thus $\mathrm{F} 555 \mathrm{~W} / \mathrm{F} 502 \mathrm{~N} \simeq 0.5$.

By contrast, a star will appear much brighter in the broad-band F555W filter than in narrow-band F502N. For a typical GC red giant, the count-rate ratios for a star will be F555W/F502N $\simeq 57$ and 30 for WFPC2 and WFC3, respectively (the WFC3 ratio being smaller because its F502N bandpass is wider than the one used in WFPC2).

At the distance of M31, the FWHM of stellar images in HST frames of $\sim 0^{\prime \prime} 06$ corresponds to a linear diameter of $\sim 0.2 \mathrm{pc}$. All but the largest PNe will appear essentially stellar at this resolution. Thus I searched the images for point sources of comparable brightness (within factors of $\sim 2$ or 0.5 , depending on the camera) in both filters, and rejected sources that were considerably brighter in F555W. I did this using two different methods: (1) First I simply visually blinked the F502N and F555W images. This technique works very well, because there are relatively few point-like sources detected in the F502N frames, especially with WFPC2, and those that are present are therefore conspicuous. 
Apart from the rare true PNe, nearly all of the apparent sources seen at F502N fell into two categories: (a) bright stars (mostly GC red giants), easily recognized because they are much brighter in the F555W frames, and (b) spurious artifacts, particularly cases where cosmic rays struck the same pixels in both of the F502N frames that were combined, sometimes producing artifacts that resemble real sources; however, these are also very easy to recognize and reject because they lack counterparts in the F555W images. (This valuable check would not have been possible if a broad-band filter had been chosen that rejected the $5007 \AA$ line.) (2) It was, however, difficult to blink the images near the centers of the GCs, because of the high surface brightness in the F555W frames. Thus I also created an image of each GC in which I calculated the ratio of the two frames, and searched them (again visually) with a display windowing that emphasized sources with similar count rates in both filters. This method worked well in the centers of the clusters.

As noted above, the target clusters were placed in the PC chip of WFPC2; however, I also searched the three neighboring WF chips, and found several new PNe in the fields surrounding the GCs, as well as recovering many previously cataloged PNe. I also searched the entire frames taken with WFC3, likewise finding a few field PNe.

Figure 1 illustrates the two search techniques. In the example shown, I independently recovered a known field PN in M31, no. 285 in the catalog of Ford \& Jacoby (1978). The left frame shows the F502N [O III] image, with the PN marked in the center, and also showing a bright star to the lower left. The middle frame shows the $V$-band F555W image; now numerous stars are also detected, and the field star is much brighter, but the PN is nearly unchanged. The right frame shows the ratio image, F502N/F555W, with the display stretched to show objects that have similar brightnesses in both frames and reject sources with low F502N/F555W ratios. Now the stars have disappeared, leaving only the PN, and verifying that it is a source emitting at $5007 \AA$. 


\subsection{Magnitude Limits}

I determined a nominal limiting magnitude for the WFPC2 F502N frames as follows. I marked apparent point-like stellar sources on a frame of the well-resolved Fornax H2 cluster over a range of brightnesses from obvious detections down to sources so faint that they were likely to be spurious. (By 2007-8, the WFPC2, which had been onboard HST since 1993, was suffering badly from CTI and hot pixels in addition to the usual cosmic-ray hits, leading to the presence of many low-level streaks and other artifacts in these frames.) I then compared the F502N frame with the F555W frame to determine which of the marked sources had brightened (indicating that they were real stars) or disappeared (indicating that they were artifacts). I found a strong cutoff at a flux level such that $100 \%$ of the sources brighter than this level were real, but below this level the fraction of spurious detections rose rapidly. I then calibrated this flux level, using aperture photometry and the reduction procedure described in the WFPC2 Data Handbook (Gonzaga \& Biretta 2010, §5.2.5). I included CTI corrections as formulated by Dolphin (2009) 3 The resulting limiting line flux for the WFPC2 frames is $F_{5007} \simeq 7.3 \times 10^{-16} \mathrm{erg} \mathrm{cm}^{-2} \mathrm{~s}^{-1}$. It is customary in work on

extragalactic PNe (e.g., Ciardullo et al. 1989) to use "[O III] $\lambda 5007$ magnitudes," defined by $m_{5007}=-2.5 \log F_{5007}-13.74$. On this scale, the source detections in my WFPC2 F502N frames are complete to $m_{5007} \simeq 24.1$. At the distance of M31, this limit reaches more than 3.5 mag into the PN luminosity function in the Andromeda bulge (e.g., Fig. 9 in JCD13).

This magnitude limit applies to isolated sources. The limit is brighter in the central regions of GCs, due to the bright backgrounds and source confusion. Using artificial-star experiments (i.e., adding an artificial PN image to the center of a GC), I find that the limiting magnitude is about 0.9 mag brighter, or about $m_{5007} \simeq 23.2$, at the center of a M31 GC of typical central surface brightness.

\footnotetext{
${ }^{3}$ Computational details are given at http://purcell.as.arizona.edu/wfpc2_calib/
} 
For WFC3, the limiting magnitudes are considerably fainter, since WFC3 is a factor of $~ 3.2$ more sensitive, has lower readout noise, and was very considerably less affected by CTI than was WFPC2 at the late stage of its lifetime. The WFC3 frames are cosmetically quite superior to the WFPC2 images, exhibiting very few low-level artifacts apart from occasional instances of a cosmic ray striking the same pixels in both F502N images. Using the same techniques, I estimated that the limiting detection magnitude for the WFC3 exposures was $m_{5007} \simeq 26.7$ for isolated sources, and again about 0.9 mag brighter for $\mathrm{PNe}$ at the centers of typical M31 GCs.

\section{Results}

\subsection{Candidates Near M31-B086}

I found only one out of the 66 GCs that were imaged in the two snapshot programs to have nearby candidate [O III] $\lambda 5007$ emission sources. I identified two sources in the vicinity of the M31 cluster B086, as shown in Figure 2. Details of these sources are given in Table 2. The brighter of them, here designated M31 B086-2, has been cataloged previously by Meyssonnier, Lequeux, \& Azzopardi (1993; no. 481 in their list of unresolved emission-line objects in M31), and by Azimlu, Marciniak, \& Barmby (2011; no. 1596 in their catalog of objects considered to be H II regions in M31).

The fainter source, M31 B086-1, has to my knowledge not been cataloged previously. Both objects appear to be unresolved at WFC3 resolution. I converted the measured count rates for both of them to $m_{5007}$ magnitudes, using the WFC3 photometric zero-points given online 4. Results are in Table 2. Since both sources are unresolved, and have values of $m_{5007}$ consistent with those of PNe in M31, it appears plausible that they are PNe (although

${ }^{4}$ http://www.stsci.edu/hst/wfc3/phot_zp_lbn 
it cannot be ruled out entirely that either one is a symbiotic binary, nova at the nebular stage, a very compact H II region, or other $5007 \AA$-emitting source). However, B086 lies in a very rich field only 4'.8 from the nucleus of M31, and fairly near a spiral arm and dust lane. Moreover, both PN candidates, at separations of 4".3 and 7".3 from the center of B086, are well outside the half-light radius of B086, measured on my images to be 0 '!8. Thus, in the absence of a spectroscopic followup and radial-velocity confirmation, it is questionable whether either object is physically associated with B086.

If the brighter source had been centered in the GC, it would have added only $3.5 \%$ to the flux of the cluster within a diameter of $1^{\prime \prime} .5$, in a bandpass similar to that of the F502N filter. The fainter source would constitute only $0.6 \%$ of the cluster flux if centered in the cluster. Thus, ground-based direct imaging would be unlikely to have detected either PN candidate if located near the cluster center. B086 was observed by JCD13, but since my PN candidates would have been well outside their spectroscopic fiber aperture, it is not surprising that they were not detected.

\section{2. $\quad$ Field Planetary Nebulae}

In the course of examining the HST frames, I noted a number of candidate $\lambda 5007$ sources in the fields surrounding the target GCs. These field sources are listed in Table 3. Although they are too distant from the GCs to be considered cluster members, I give them designations based on the name of the target cluster. As an index of my survey completeness, I include previously cataloged objects that I independently recovered, as well as new discoveries. The table includes $m_{5007}$ values measured from my material; I adjusted the zero-point for these magnitudes so as to match the absolute line fluxes listed by Ciardullo et al. (1989) for the sources in common. The rms scatter was 0.2 mag, consistent with the moderately low SNR of the WFPC2 [O III] snapshot images. 
The final column in Table 3 gives cross references for the previously known sources. Nearly all of the sources brighter than $m_{5007} \simeq 23.5$ have been cataloged previously. All of the listed objects appear stellar at HST resolution, making it likely that most of them are PNe, even though some were included in earlier compilations of H II regions. However, one object appears to coincide with a classical nova.

\section{3. $\quad$ Searching the $H S T$ Archive}

I augmented my snapshot observations by searching the HST archive 5 for frames taken by other investigators with any of the HST cameras, using a [O III] F502N filter, in the vicinity of M31, and then checking whether any GCs were within the fields of view. Surprisingly, only two HST programs, other than my own, have ever used this filter at pointings lying within $3^{\circ}$ of the center of M31. Fortuitously, 12 cataloged GCs lie within the fields observed in these two programs. Images were obtained with the Advanced Camera for Surveys (ACS) or WFC3, as listed in Table 4. Three of these clusters had also been imaged in my snapshot survey, so the grand total number of Local Group GCs outside the Milky Way imaged by HST in a F502N filter is 75 .

The F502N images in the two archival programs were accompanied either by frames in F547M or F550M (which exclude the $5007 \AA$ emission line) or in F555W (which includes $5007 \AA$ as described above). Out of the 12 clusters, one very convincing PN candidate was found within the M31 cluster B477, as illustrated in Figure 3. Details are given in Table 4. This emission-line object had been listed by Walterbos \& Braun (1992; no. 683, which they described as being unresolved, in their catalog of $\mathrm{H} \alpha /[\mathrm{S} \mathrm{II}]$ sources in M31), and by Merrett et al. (2006, hereafter MMD06; no. 446 in their catalog of PNe in M31). The $m_{5007}$

\footnotetext{
${ }^{5}$ http://archive.stsci.edu/hst/search.php
} 
value in Table 4 is quoted from MMD06. M31-B477 was not one of the clusters observed spectroscopically by JCD13, and the association with the star cluster has apparently not been recognized until now. B477 is, however, not considered to be an old GC of the type targeted in my snapshot survey: Kang et al. (2012) give an age of only 325 Myr, based on Galaxy Evolution Explorer UV photometry. Morphologically, B477 also does not resemble a GC, as can be seen in Figure 3.

\section{Comparison with JCD13}

The present imaging survey is complementary in several ways to the ground-based spectroscopic search for PNe in the M31 GCs carried out by JCD13. My survey concentrated on the brightest GCs in M31 (and the rest of the Local Group), while their large-scale spectroscopic program targeted a wide range of cluster luminosities in M31. Their method is particularly favorable for PNe in fainter GCs, in which there is less dilution of the PN $5007 \AA$ emission by cluster starlight in the integrated spectrum. Their spectroscopic apertures had diameters of $3^{\prime \prime}$, which captures most of the cluster light but would still miss more widely separated PNe, whereas I covered the entire cluster plus a large surrounding field. JCD13 observed 274 old GCs in M31, as compared to only 75 imaged in my survey (plus two archival programs) throughout the Local Group. Of the 56 GCs in M31 that I observed or had archival F502N images, JCD13 obtained spectra of 39 of them. JCD13 were able to reach fainter limiting magnitudes than my WFPC2 frames, down to about $m_{5007} \simeq 25.9$ vs. my 24.1 , because they used a larger telescope (3.5m vs. $\left.2.4 \mathrm{~m}\right)$, and much longer integration times (210 minutes vs. 600-1000 s). The limiting magnitudes for my WFC3 frames are, however, similar to those of JCD13. The spectroscopic observations are susceptible to spurious detections of ambient [O III] emission from diffuse nebulae superposed on the GC, whereas direct high-resolution imaging of point sources does not 
suffer from this problem.

One inadequacy of direct imaging in [O III $]$ is that it does not provide a line profile, line ratios such as $\left[\begin{array}{ll}\mathrm{O} & \mathrm{III}\end{array}\right] / \mathrm{H} \beta$, or other spectroscopic information, all of which help discriminate PNe from other emission-line sources. For example, Zepf et al. (2008) found very broad [O III] emission from a source within a GC in the Virgo galaxy M49, attributed to an accreting black hole $(\mathrm{BH})$ rather than a PN. Irwin et al. (2010) found [O III] emission, unaccompanied by Balmer emission, from a GC in the Fornax galaxy NGC 1399; they discussed this source in terms of a white-dwarf being tidally disrupted by an intermediate-mass $\mathrm{BH}$, and alternative non-PN scenarios for this object have been proposed by Maccarone \& Warner (2011) and Clausen et al. (2012).

JCD13 found candidate PNe in three old GCs in M31: NB89, B115, and BH16. Of these, only NB89 was observed in my snapshot programs, and only because it happens to lie a few arcseconds away from the bright primary target GC B127. Much deeper HST [O III] imaging of NB89 - two exposures of $2700 \mathrm{~s}$ each — was obtained in the archival WFC3 program GO-12174, as summarized in Table 4. The PN candidate in NB89 has $m_{5007}=25.1$ according to JCD13. An isolated point source this bright would have been detected easily in the deep WFC3 frames, yet no $5007 \AA$ point source is seen in the images. It is conceivable that the PN coincides exactly with a red giant in the cluster. However, a more likely possibility is that the emission arises from ambient nebular emission that filled JCD13's aperture, rather than from a point source - indeed, JCD13 raised this as a strong possibility. In fact, NB89 does lie exactly superposed on a filament of the spiral-shaped emission region discovered by Ciardullo et al. (1988) in their deep ground-based H $\alpha$ imaging of the M31 nucleus. Although it is unclear whether this low-excitation region has sufficiently strong $5007 \AA$ emission for this explanation, the lack of a point-like source in the HST [O III] images supports this view. 
There is no available narrow-band $5007 \AA$ HST imaging for the other two clusters, B115 and BH16, in which JCD13 detected [O III] emission, with $m_{5007}$ values of 22.6 and 25.3, respectively. However, there are archival broad-band optical and near-UV images for

both clusters, obtained in the Andromeda Treasury program GO-12058 (PI J. Dalcanton). Veyette et al. (2014) have shown that PNe have distinctive optical and NUV broad-band colors. This is especially true shortward of the Balmer jump, where there are significant contributions to the flux from the nebular continuum and numerous emission lines, and from the underlying photosphere of the central star.

In Figure 4 I show HST images of B115 and BH16, taken with WFC3 in the near UV (F275W) and optical UV (F336W), and with ACS at approximately the $B$ band (F475W). The sizes of the frames are similar to those of the spectroscopic apertures used by JCD13. In both cases, there is a conspicuous, very blue star-like object within the field. It is probable that these are the PNe detected by JCD13, although high-resolution narrow-band imaging would be needed to absolutely confirm the identifications. Similar sets of broad-band WFC3 and ACS images exist for NB89, but in this case there is no obvious blue star in or near the cluster. This is consistent with the above suggestion that the [O III] emission in NB89 is from ambient diffuse nebulosity rather than a PN.

\section{Intercomparison of M31 Cluster and PN Catalogs}

The current online version of the Galleti et al. (2004) M31 catalog (version 5, 2012 August) has 2060 entries, which include confirmed GCs, candidate GCs, and candidate objects subsequently shown not to be genuine GCs. The MMD06 catalog of PNe in M31 contains 3300 confirmed PNe and PN candidates. I intercompared these two catalogs, finding 44 cases where the coordinates agreed within $4^{\prime \prime}$. A majority of these objects are actually candidate GCs that have been reclassified as H II regions by Caldwell et al. (2009), 
and are thus neither GCs nor PNe. Sixteen of the GCs have not been classified; most of them are from the catalog of Kim et al. (2007), and I suspect that they are also misclassified H II regions.

Of these 44 clusters, only seven were included in the spectroscopic observations of JCD13 (B075, B105, BH16, B530, SK052A, SK070A, and SK104A). JCD13 reported $5007 \AA$ emission only in the old cluster BH16 (see $\S 5$ above). For the other six, the nominal separations of the emission-line objects from the clusters are larger than the JCD13 aperture radius.

There are five cases where an M31 cluster classified as an "old" GC by Caldwell et al. (2009, 2011), Peacock et al. (2010), Strader et al. (2011), and/or JCD13 has an MMD06 source lying within $4^{\prime \prime}$. These are listed in Table 6 . Of these five, there are archival $H S T$ images available for only two. (1) BH16 was discussed in the previous section, in which I suggested that a blue point-like source to the SE of the cluster center, which would have been within JCD13's spectroscopic aperture, is the PN. Curiously, however, the PN detected by JCD13 does not appear to be the object cataloged as MMD06 1360, which differs substantially in both radial velocity and $m_{5007}$ from the JCD13 PN. The HST images show a bright blue star lying 3".2 WSW of the cluster, which may be the MMD06 source. (2) SK104A also has a neighboring blue star in broad-band HST images, lying 1".2 NNE from the cluster center. This is possibly the PN candidate, but narrow-band imaging and/or spectroscopy is needed to confirm this 6

\footnotetext{
${ }^{6} \mathrm{~A}$ similar intercomparison of cataloged star clusters and PNe in the LMC was made by Kontizas et al. 1996; of the four dozen possible associations that they identified, none lie near the old LMC GCs listed by Olszewski et al. 1996.
} 


\section{Summary and Discussion}

I used HST snapshot observing to obtain [O III] $5007 \AA$ images of 55 of the brightest "Population II" GCs in Local Group galaxies outside the Milky Way. An additional 11 fainter clusters serendipitously fell within these frames. A search of the $H S T$ archive found [O III] images of nine more GCs in M31, for a grand total of 75 Local-Group GCs. I searched all 75 of these clusters for point-like $5007 \AA$ sources, which would be strong PN candidates.

Among these old GCs of the Local Group, I found only the M31 cluster B086 to have two PN candidates in its vicinity. However, they are at such large angular separations that their cluster membership is doubtful. One very convincing PN candidate was found in images of the young M31 cluster B477.

I also investigated HST images of the three candidate PNe found in old clusters by JCD13 in their ground-based spectroscopic survey of M31 GCs. One of them appears to be a case where ambient diffuse nebulosity produced the $5007 \AA$ emission, rather than a true PN. For the other two, there are only broad-band HST images available, but both clusters contain a bright blue point source which is plausibly the PN. A comparison of two extensive catalogs of GCs and PNe in M31 revealed several additional candidate PNe within a few arcseconds of old GCs, but spectroscopy and narrow-band HST images will be needed to verify the cluster membership.

The total luminosity of the 157 known GCs in the Milky Way system, determined from the data in the Harris (1996) compliation (2010 edition?]), corresponds to a visual absolute magnitude of $M_{V}=-13.3$. For my sample of 75 Local-Group GCs, although only numbering about half of the Milky Way clusters, the total luminosity is actually slightly

\footnotetext{
${ }^{7}$ available at http://www.physics.mcmaster.ca/ harris/mwgc.dat
} 
higher, at $M_{V}=-13.6$.

In the Milky Way GC system, there are four known PNe (JMF97; Table 4 of JCD13). If they were at the distance of M31, they would have $m_{5007}$ magnitudes of 21.3, 25.3, 27.4, and 29.7. Given the limiting magnitudes of my surveys (§3.2), only one of them would be bright enough to be detected in my WFPC2 snapshots, and one more in my deeper WFC3 frames. In the actual old-population M31 clusters, we have two very probable $\mathrm{PNe}$ - those in B115 and BH16 - along with several more candidates ( $\S 4.1,4.3)$, which are as yet unconfirmed. As noted in $\S 1$, there is also a PN in one of the GCs of the Fornax dwarf spheroidal (Larsen 2008). Thus, within the small-number statistics, the incidences of PNe in the old GCs of the Milky Way and in the rest of the Local Group are very similar.

According to our understanding of stellar evolution, we do not expect that PNe can be formed by single stars in the ancient populations of GCA 8 . Binary-star mergers and CE events provide a plausible explanation for the fact that a few PNe nevertheless exist in these clusters. One observational test would be to search for evidence of binarity in the central stars of PNe in Milky Way GCs: those whose masses appear to be too low to have resulted from mergers are likely to have gone through a CE interaction, leaving them as close but still un-merged binaries.

I also found some 60 point-like [O III] $5007 \AA$ sources in the fields surrounding the

\footnotetext{
${ }^{8}$ However, two of the PNe associated with GCs appear to be hydrogen-deficient: IRAS 18333-2357 in M22 (Gillett et al. 1989) and the PN in the Fornax GC H5 (Larsen 2008). This may raise the possibility for these two objects of a late thermal pulse (in a single star), similar to that invoked to explain the compact H-deficient [O III]-emitting nebula surrounding V605 Aquilae (Clayton et al. 2013 and references therein). Detailed discussion is given by Jacoby et al. (2015)
} 
GCs (all but one of them in M31); many of them were already cataloged, but the stellar appearances in the HST images verify that they are highly probable field PNe.

Support for program numbers SNAP-11218 and SNAP-11714 was provided by NASA through grants from the Space Telescope Science Institute, which is operated by the Association of Universities for Research in Astronomy, Incorporated, under NASA contract NAS5-26555. Helpful assistance and advice on the HST scheduling was given by Alison Vick, Denise Taylor, Sylvia Baggett, Ronald Gilliland, and Larry Petro. This research has made extensive use of the SIMBAD database, operated at CDS, Strasbourg, France; and of the catalogs of Galleti et al. (2004), Merrett et al. (2006), and Harris (1996).

Facilities: HST (ACS, WFPC2, WFC3) 


\section{REFERENCES}

Alves, D. R., Bond, H. E., \& Livio, M. 2000, AJ, 120, 2044 (ABL00)

Azimlu, M., Marciniak, R., \& Barmby, P. 2011, AJ, 142, 139

Bond, H. E. 2000, Asymmetrical Planetary Nebulae II: From Origins to Microstructures, 199,115

Bond, H. E., \& Livio, M. 1990, ApJ, 355, 568

Bond, H. E., \& Quijano, J. K. 2007, WFC3 Instrument Handbook, Version 1.0 (Baltimore: STScI)

Buell, J. F. 2012, MNRAS, 419, 2867

Caldwell, N., Harding, P., Morrison, H., et al. 2009, AJ, 137, 94

Caldwell, N., Schiavon, R., Morrison, H., Rose, J. A., \& Harding, P. 2011, AJ, 141, 61

Christian, C. A., \& Schommer, R. A. 1988, AJ, 95, 704

Ciardullo, R., Jacoby, G. H., Ford, H. C., \& Neill, J. D. 1989, ApJ, 339, 53

Ciardullo, R., Rubin, V. C., Ford, W. K., Jr., Jacoby, G. H., \& Ford, H. C. 1988, AJ, 95, 438

Ciardullo, R., Sigurdsson, S., Feldmeier, J. J., \& Jacoby, G. H. 2005, ApJ, 629, 499

Clausen, D., Sigurdsson, S., Eracleous, M., \& Irwin, J. A. 2012, MNRAS, 424, 1268

Clayton, G. C., Bond, H. E., Long, L. A., et al. 2013, ApJ, 771, 130

De Marco, O. 2009, PASP, 121, 316

Dolphin, A. E. 2009, PASP, 121, 655

Ford, H. C., \& Jacoby, G. H. 1978, ApJS, 38, 351

Ford, H. C., Jacoby, G., \& Jenner, D. C. 1977, ApJ, 213, 18 
Galleti, S., Federici, L., Bellazzini, M., Fusi Pecci, F., \& Macrina, S. 2004, A\&A, 416, 917

Gillett, F. C., Jacoby, G. H., Joyce, R. R., et al. 1989, ApJ, 338, 862

Gonzaga, S., \& Biretta, J. 2010, HST WFPC2 Data Handbook, Version 5.0 (Baltimore: $\mathrm{STScI})$

Hannikainen, D. C., Charles, P. A., van Zyl, L., et al. 2005, MNRAS, 357, 325

Harris, W. E. 1996, AJ, 112, 1487

Hodge, P. W., Dolphin, A. E., Smith, T. R., \& Mateo, M. 1999, ApJ, 521, 577

Hurley-Keller, D., Morrison, H. L., Harding, P., \& Jacoby, G. H. 2004, ApJ, 616, 804

Irwin, J. A., Brink, T. G., Bregman, J. N., \& Roberts, T. P. 2010, ApJ, 712, L1

Jacoby, G. H., Ciardullo, R., De Marco, O., et al. 2013, ApJ, 769, 10 (JCD13)

Jacoby, G. H., De Marco, O., Davies, J. E., Harrington, J. P., \& Bond, H. E. 2014, American Astronomical Society Meeting Abstracts \#223, 223, \#353.25

Jacoby, G. H., De Marco, O., Davies, J., et al. 2015, in preparation

Jacoby, G. H., Morse, J. A., Fullton, L. K., Kwitter, K. B., \& Henry, R. B. C. 1997, AJ, 114, 2611 (JMF97)

Kalirai, J. S., Saul Davis, D., Richer, H. B., et al. 2009, ApJ, 705, 408

Kang, Y., Rey, S.-C., Bianchi, L., et al. 2012, ApJS, 199, 37

Kim, S. C., Lee, M. G., Geisler, D., et al. 2007, AJ, 134, 706

Kontizas, M., Morgan, D. H., Kontizas, E., \& Dapergolas, A. 1996, A\&A, 307, 359

Larsen, S. S. 2008, A\&A, 477, L17

Maccarone, T. J., \& Warner, B. 2011, MNRAS, 410, L32

McMaster, M., \& Biretta, J. 2008, WFPC2 Instrument Handbook, Version 10.0 (Baltimore: $\mathrm{STScI})$ 
Merrett, H. R., Merrifield, M. R., Douglas, N. G., et al. 2006, MNRAS, 369, 120 (MMD06)

Meyssonnier, N., Lequeux, J., \& Azzopardi, M. 1993, A\&AS, 102, 251

Minniti, D., \& Rejkuba, M. 2002, ApJ, 575, L59

Olszewski, E. W., Suntzeff, N. B., \& Mateo, M. 1996, ARA\&A, 34, 511

Otsuka, M., Tajitsu, A., Hyung, S., \& Izumiura, H. 2010, ApJ, 723, 658

Peacock, M. B., Maccarone, T. J., Knigge, C., et al. 2010, MNRAS, 402, 803

Peacock, M. B., Zepf, S. E., \& Maccarone, T. J. 2012, ApJ, 752, 90

Pease, F. G. 1928, PASP, 40, 342

Renzini, A., \& Buzzoni, A. 1986, Spectral Evolution of Galaxies, 122, 195

Schoenberner, D. 1983, ApJ, 272, 708

Sharina, M. E., Afanasiev, V. L., \& Puzia, T. H. 2006, MNRAS, 372, 1259

Strader, J., Brodie, J. P., Forbes, D. A., Beasley, M. A., \& Huchra, J. P. 2003, AJ, 125, 1291

Strader, J., Caldwell, N., \& Seth, A. C. 2011, AJ, 142, 8

van den Bergh, S. 2010, AJ, 140, 1043

Veyette, M. J., Williams, B. F., Dalcanton, J. J., et al. 2014, ApJ, 792, 121

Walterbos, R. A. M., \& Braun, R. 1992, A\&AS, 92, 625

Wyder, T. K., Hodge, P. W., \& Zucker, D. B. 2000, PASP, 112, 1162

Zepf, S. E., Stern, D., Maccarone, T. J., et al. 2008, ApJ, 683, L139 


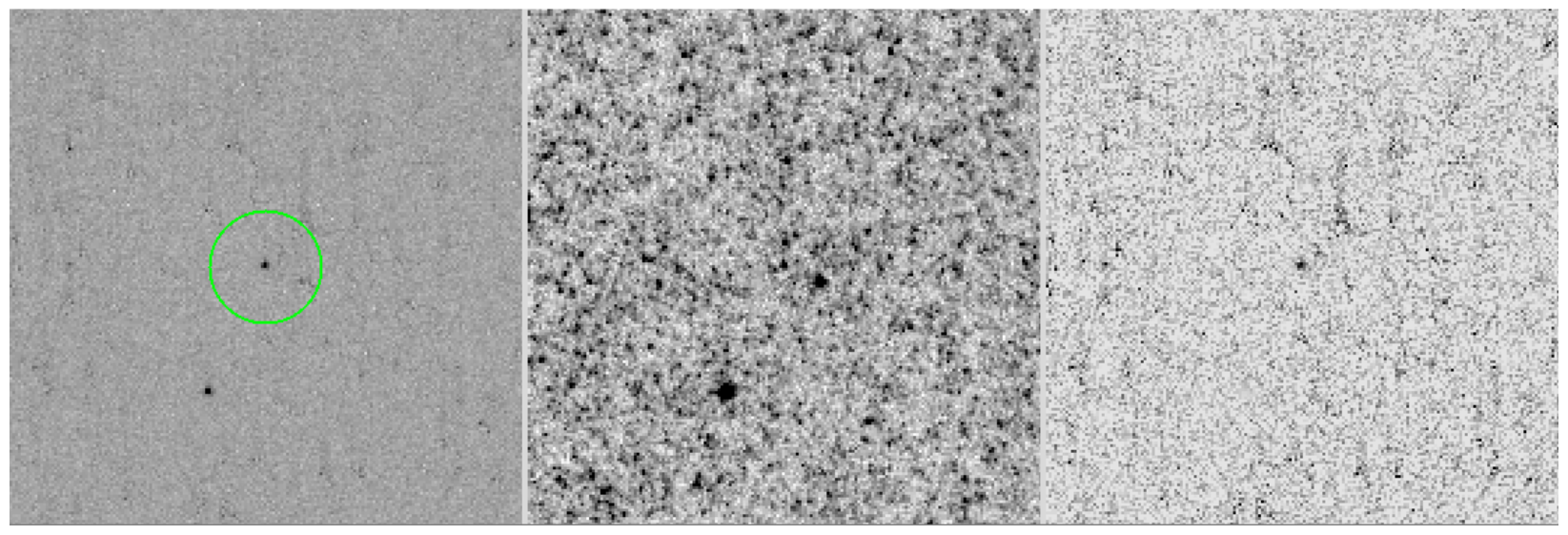

Fig. 1.- Illustration of PN search techniques. These are HST WFPC2/WF2 images of planetary nebula no. 285 (Ford \& Jacoby 1978) in a field in M31. Frames are 17".9 high. Left: Image in F502N ([O III] $5007 \AA$ ); the PN is circled in green. Middle: Image in F555W (broad-band $V$ ). Now numerous stars are detected, and the star to the lower left of the $\mathrm{PN}$ is much brighter. However, the PN has approximately the same counts as in F502N. Right: Ratio image F502N/F555W, windowed to emphasize objects with similar counts in both filters. The stars are not seen, including the bright one to the lower left of the nebula, leaving only the PN plus a number of faint cosmetic artifacts. Faint vertical streaks are due to charge-transfer inefficiency in the aging WFPC2 detector. 


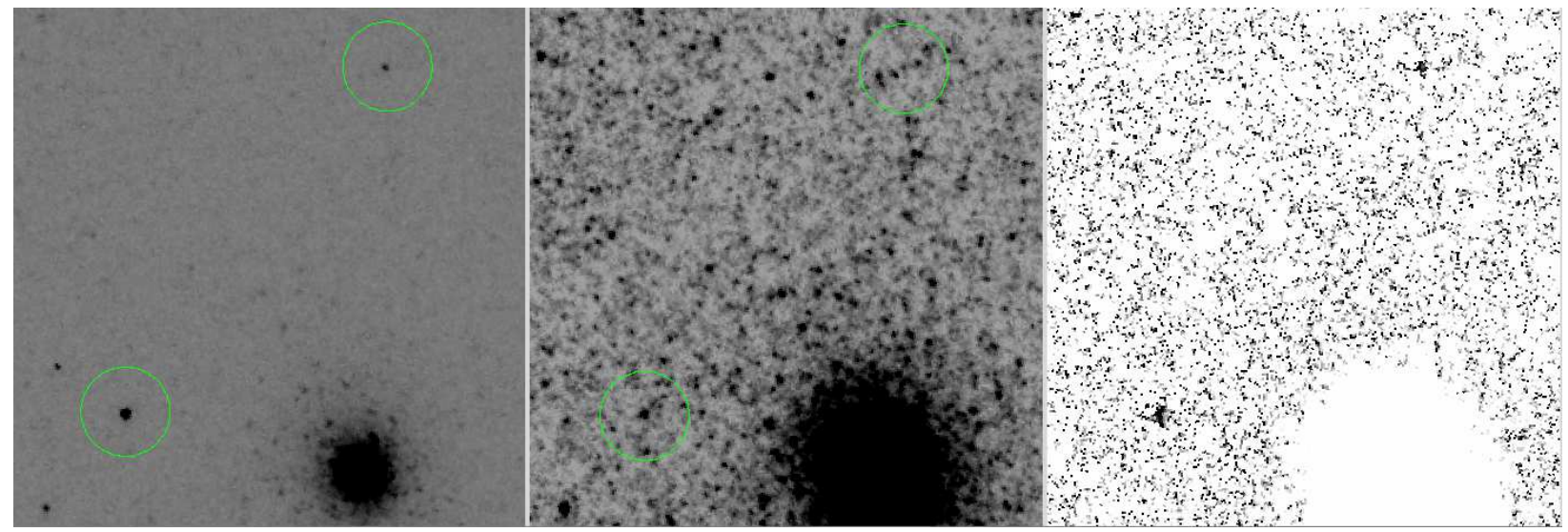

Fig. 2.- HST WFC3 images showing two candidate [O III $] 5007 \AA$ emission-line PNe near the M31 globular cluster B086. Frames are 9'.2 high and have north at the top, east at the left. Left: Image in F502N ([O III] $5007 \AA$ ); the two sources are circled in green. Middle: Image in F555W (broad-band V). Right: Ratio image F502N/F555W, windowed to emphasize objects with similar count rates in both filters and reject stellar sources with low $[\mathrm{O}$ III $] /$ continuum ratios. 


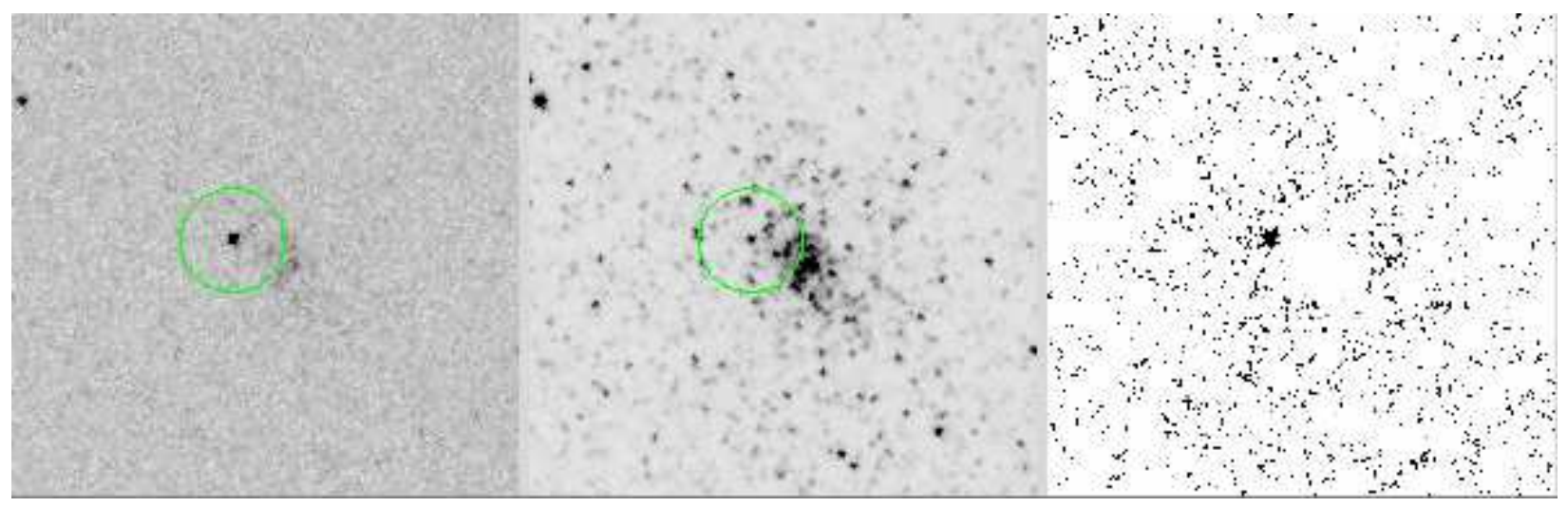

Fig. 3.- Archival HST ACS images showing the PN in the M31 young star cluster B477. Frames are 9!'2 high and have north at the top, east at the left. Left: Image in F502N ([O III] $5007 \AA$ ); the $\mathrm{PN}$ is circled in green. Middle: Image in F555W (broad-band $V$ ). Right: Ratio image F502N/F555W, windowed to emphasize objects with similar count rates in both filters and reject stellar sources with low $[\mathrm{O} \mathrm{III}] /$ continuum ratios. 


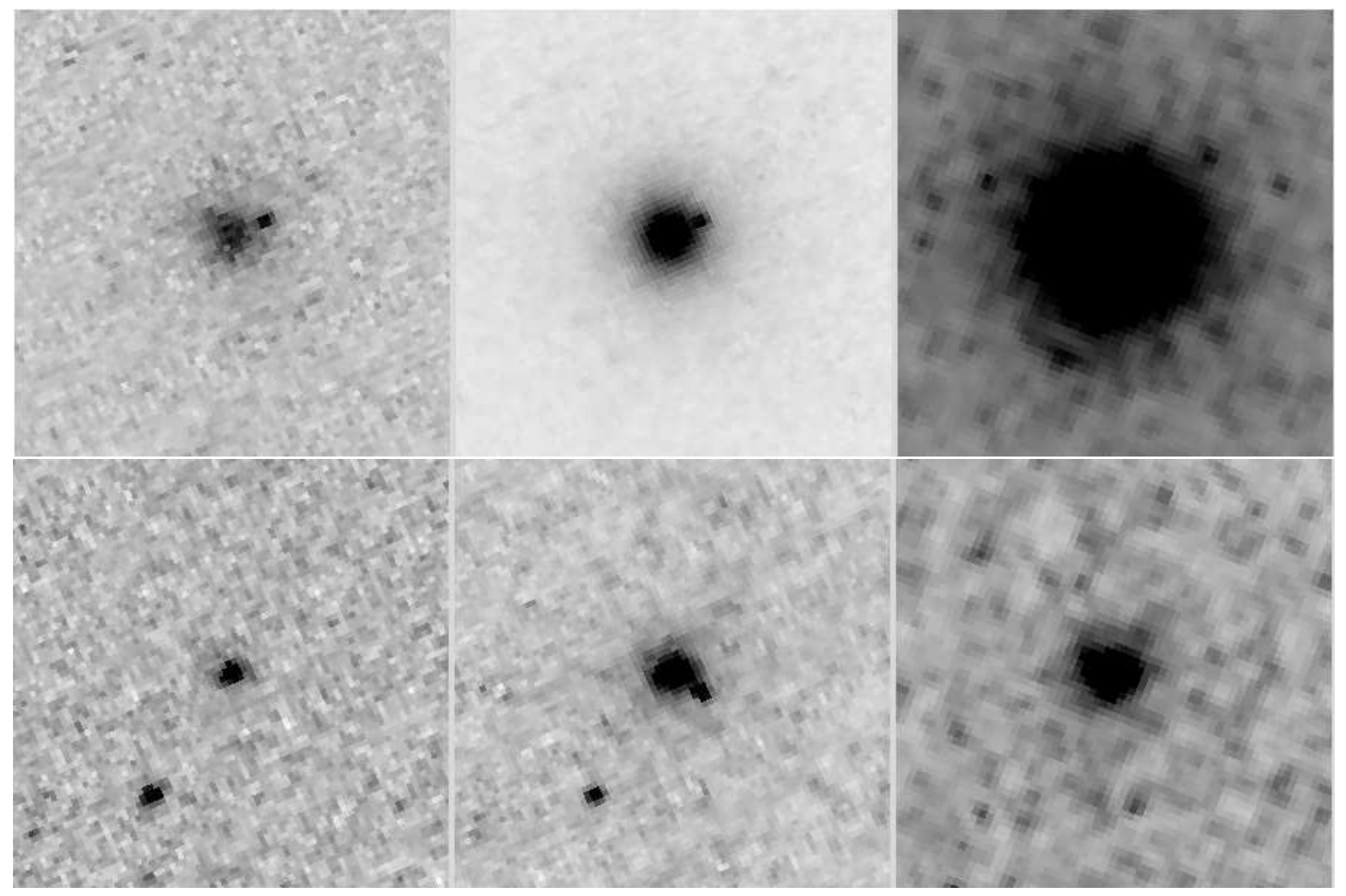

Fig. 4.- HST WFC3 and ACS broad-band images of the M31 globular clusters B115 (top row) and BH16 (bottom row), each of which contains a PN candidate found by Jacoby et al. (2013). Frames are 2".9 high and have north at the top, east at the left. Left: WFC3 images in F275W. Middle: WFC3 images in F336W. Right: ACS images in F475W, dominated by red giants and horizontal-branch stars in the cluster. For B115, both of the UV images show a bright blue stellar source 0".22 NW of the cluster center, which is probably the central star of the PN. For BH16, both UV images show a bright blue stellar source 0'.98 SE of the cluster center, which is also probably the PN nucleus. The BH16 image in F336W also shows a cosmic-ray artifact to the immediate SW of the cluster center. 
Table 1. HST Snapshot Imaging of Local-Group Globular Clusters

\begin{tabular}{|c|c|c|c|}
\hline Galaxy \& Cluster & $M_{V}^{\mathrm{a}}$ & WFPC2 Date & WFC3 Date \\
\hline Fornax: H2 & -7.2 & $2008-11-23$ & 2010-01-10 \\
\hline Fornax: NGC 1049 & -8.1 & 2008-09-19 & 2010-02-01 \\
\hline LMC: NGC 1466 & -7.9 & & 2009-09-01 \\
\hline LMC: NGC 1786 & -7.9 & 2008-09-19 & $2010-02-06$ \\
\hline LMC: NGC 1835 & -9.2 & $2007-08-28$ & \\
\hline LMC: NGC 1916 & -9.0 & 2008-06-01 & \\
\hline LMC: NGC 2005 & -7.5 & 2008-11-22 & 2009-12-05 \\
\hline LMC: NGC 2019 & -7.9 & $2008-08-26$ & $2009-10-28$ \\
\hline LMC: NGC 2210 & -8.1 & $2007-12-26$ & \\
\hline M31-B005 & -9.0 & 2008-11-15 & \\
\hline M31-B008 & -7.8 & & 2010-01-02 \\
\hline M31-B019 & -9.6 & $2008-08-23$ & \\
\hline M31-B023 & -10.4 & 2008-08-05 & 2011-09-05 \\
\hline M31-B027 & -9.0 & $2007-08-28$ & \\
\hline M31-B037 & -7.8 & 2007-09-13 & \\
\hline M31-B041 & -6.0 & 2007-09-13 & \\
\hline M31-B058 & -9.6 & $2008-07-22$ & \\
\hline M31-B061 b & -8.0 & $2007-07-17$ & \\
\hline M31-B063 & -8.9 & 2007-07-17 & \\
\hline M31-B082 & -9.0 & 2008-07-21 & \\
\hline M31-B086 & -9.4 & & 2011-01-26 \\
\hline M31-B097D & -6.2 & 2008-07-28 & \\
\hline M31-B103D & -6.9 & 2008-09-09 & \\
\hline M31-B110 & -9.3 & 2008-11-15 & \\
\hline M31-B124 b & -9.8 & 2008-11-16 & \\
\hline
\end{tabular}


Table 1-Continued

\begin{tabular}{|c|c|c|c|}
\hline Galaxy \& Cluster & $M_{V}^{\mathrm{a}}$ & WFPC2 Date & WFC3 Date \\
\hline M31-B127 & -10.1 & $2008-11-16$ & \\
\hline M31-B158 & -9.9 & $2008-07-28$ & \\
\hline M31-B171 & -9.4 & & 2011-09-29 \\
\hline M31-B174 & -9.1 & 2008-11-15 & \\
\hline M31-B179 & -9.2 & 2008-08-10 & \\
\hline M31-B192 & -6.4 & 2008-11-15 & \\
\hline M31-B193 & -9.2 & 2008-11-15 & \\
\hline M31-B225 & -10.4 & 2008-06-16 & \\
\hline M31-B293 & -8.3 & 2007-09-24 & \\
\hline M31-B311 & -9.1 & 2008-06-19 & \\
\hline M31-B312 & -9.0 & $2008-11-17$ & 2010-09-03 \\
\hline M31-B327 ${ }^{\mathrm{b}}$ & -8.1 & 2007-09-12 & \\
\hline M31-B338 & -10.3 & 2008-06-19 & \\
\hline M31-B343 & -8.3 & & 2011-08-14 \\
\hline M31-B366 & -8.6 & & 2010-09-04 \\
\hline M31-B373 & -8.9 & 2008-07-24 & \\
\hline M31-B379 & -8.4 & 2007-09-30 & \\
\hline M31-B384 & -8.8 & & 2009-10-09 \\
\hline M31-B386 & -9.0 & $2008-07-28$ & \\
\hline M31-B405 & -9.4 & 2008-06-14 & \\
\hline M31-B407 & -8.5 & 2008-06-18 & \\
\hline M31-B472 & -9.4 & 2008-09-09 & \\
\hline M31-B480 & -6.7 & 2008-07-24 & \\
\hline M31-B514 & -8.8 & 2007-07-19 & \\
\hline M31-G001 & -10.8 & 2007-07-16 & \\
\hline
\end{tabular}


Table 1-Continued

\begin{tabular}{llll}
\hline \hline & & & \\
Galaxy \& Cluster & $M_{V}^{\mathrm{a}}$ & WFPC2 Date & WFC3 Date \\
\hline & & & \\
M31-G002 & -8.8 & $2008-09-24$ & \\
M31-MCGC1 & -8.5 & & $2009-09-30$ \\
M31-MCGC3 & -8.3 & & $2009-11-09$ \\
M31-MCGC5 & -8.4 & & $2009-12-02$ \\
M31-NB89 & -6.6 & $2008-11-16$ & \\
M31-VDB0 & -9.8 & $2007-09-12$ & \\
M33-MKK33 & -8.1 & & $2011-06-16$ \\
M33-R12 & -8.1 & & $2009-12-02$ \\
M33-R14 & -8.0 & & $2010-02-26$ \\
M33-SM310 & -6.1 & & $2009-12-02$ \\
M33-U49 & -8.2 & & \\
NGC 147: Hodge II & -7.4 & $2008-11-21$ & \\
NGC 147: Hodge III & -6.9 & $2008-11-21$ & \\
NGC 6822: H VII & -7.7 & $2007-07-10$ & \\
SMC: NGC 121 & -7.8 & $2007-11-19$ & \\
WLM: WLM-1 & -8.8 & $2008-09-08$ & \\
\hline
\end{tabular}

${ }^{a}$ Absolute integrated visual magnitude, calculated from distance and reddening of each galaxy and the integrated apparent $V$ magnitude

${ }^{\mathrm{b}}$ The following faint clusters were observed serendipitously in the WFPC2 fields of the primary targets given in parentheses: M31-B041 (M31-B037); M31-B061 (M31-B063); M31B097D (M31-B158); M31-B103D (M31-B472); M31-B124 (M31- 
B127); M31-B192 (M31-B193); M31-B327 (M31-B195D); M31B480 (M31-B373); M31-NB89 (M31-B127); NGC 147 Hodge III (NGC 147 Hodge II).

'The following faint cluster was observed serendipitously in the WFC3 field of the primary target given in parentheses: M33SM310 (M33-R12). 
Table 2. Candidate Planetary Nebulae Near M31 Cluster B086

\begin{tabular}{lllll}
\hline \hline & & & & \\
Designation & RA (J2000) & Dec. (J2000) & $r^{\mathrm{a}}$ & $m_{5007}$ \\
\hline & & & & \\
M31 B086-1 & $00: 42: 18.62$ & $41: 14: 08.8$ & $7^{\prime \prime} .3$ & 24.1 \\
M31 B086-2 & $00: 42: 19.03$ & $41: 14: 02.6$ & $4^{\prime \prime} .3$ & 22.1 \\
\hline
\end{tabular}

${ }^{a}$ Angular distance from cluster center 
Table 3. Field Candidate Planetary Nebulae

\begin{tabular}{|c|c|c|c|c|}
\hline Designation & RA (J2000) & Dec. (J2000) & $m_{5007}$ & Cross reference ${ }^{\mathrm{a}}$ \\
\hline NGC 147 HII-1 & $00: 33: 12.62$ & $48: 28: 17.5$ & 22.6 & FJJ NGC 147-1 \\
\hline M31 B005-1 & 00:40:16.29 & $40: 43: 23.2$ & 24.0 & AMB 551; MMD 1944 \\
\hline M31 B195D-1 & $00: 40: 25.75$ & $40: 37: 06.0$ & 23.2 & MMD 2052 \\
\hline M31 B195D-2 & $00: 40: 30.82$ & $40: 36: 53.8$ & 20.7 & MLA 47; HKPN 47; MMD 2049 \\
\hline M31 B023-1 & $00: 40: 58.27$ & $41: 14: 16.8$ & 23.5 & \\
\hline M31 B037-1 & $00: 41: 37.51$ & $41: 15: 10.6$ & 22.2 & MLA 378; MMD 1052 \\
\hline M31 B063-1 & 00:42:06.30 & $41: 29: 29.5$ & 24.0 & \\
\hline M31 B086-3 & $00: 42: 16.99$ & 41:14:02.3 & 24.6 & \\
\hline M31 B082-1 & $00: 42: 17.84$ & 41:01:15.6 & 22.4 & MLA 485 \\
\hline M31 B127-1 & $00: 42: 37.07$ & $41: 14: 34.8$ & 20.6 & FJ 54; MMD 2811 \\
\hline M31 B127-2 & $00: 42: 37.47$ & $41: 14: 34.3$ & 21.9 & FJ 71; MMD 2826 \\
\hline M31 B127-3 & $00: 42: 38.26$ & $41: 15: 33.4$ & 21.4 & FJ 35; MMD 2815 \\
\hline M31 B127-4 & $00: 42: 38.36$ & $41: 14: 26.9$ & 22.6 & FJ 143; MMD 2827 \\
\hline M31 B127-5 & $00: 42: 38.38$ & $41: 14: 33.9$ & 21.3 & FJ 55; MMD 2810 \\
\hline M31 B127-6 & $00: 42: 38.95$ & $41: 14: 56.0$ & 21.9 & FJ 70; MMD 2812 \\
\hline M31 B127-7 & 00:42:39.39 & $41: 14: 17.1$ & 22.1 & FJ 65; MMD 2809 \\
\hline M31 B127-8 & 00:42:39.72 & $41: 15: 36.3$ & 21.0 & FJ 18; MMD 2817 \\
\hline M31 B127-9 & $00: 42: 39.75$ & $41: 15: 49.3$ & 20.9 & FJ 17; MMD 2825 \\
\hline M31 B127-10 & 00:42:40.08 & $41: 14: 37.8$ & 22.0 & FJ 66; MMD 2828 \\
\hline M31 B127-11 & $00: 42: 40.12$ & $41: 13: 23.9$ & 22.8 & FJ 159; MMD 2822 \\
\hline M31 B127-12 & $00: 42: 40.18$ & $41: 15: 32.7$ & 23.1 & FJ 334 \\
\hline M31 B127-13 & $00: 42: 40.20$ & $41: 13: 50.3$ & 23.2 & FJ 160; MMD 2824 \\
\hline
\end{tabular}


Table 3-Continued

\begin{tabular}{|c|c|c|c|c|}
\hline Designation & RA (J2000) & Dec. (J2000) & $m_{5007}$ & Cross reference $^{\mathrm{a}}$ \\
\hline M31 B127-14 & $00: 42: 40.28$ & $41: 15: 44.2$ & 23.3 & FJ 333 \\
\hline M31 B127-15 & $00: 42: 40.34$ & 41:14:09.5 & 22.0 & FJ 181; MMD 2823 \\
\hline M31 B127-16 & 00:42:40.69 & 41:14:09.4 & 20.9 & FJ 56; MMD 1250 \\
\hline M31 B127-17 & $00: 42: 41.34$ & 41:14:06.9 & 22.1 & FJ 141; MMD 1251 \\
\hline M31 B127-18 & 00:42:42.10 & 41:14:09.0 & 21.5 & FJ 57; MMD 1249 \\
\hline M31 B127-19 & $00: 42: 42.15$ & $41: 14: 22.3$ & 22.6 & FJ 174; MMD 1255 \\
\hline M31 B127-20 & $00: 42: 42.33$ & $41: 15: 53.3$ & 21.1 & FJ 4; MMD 1339 \\
\hline M31 B127-21 & $00: 42: 42.43$ & $41: 13: 55.9$ & 22.1 & FJ 99; MMD 1246 \\
\hline M31 B127-22 & 00:42:42.62 & $41: 13: 33.8$ & 23.9 & \\
\hline M31 B127-23 & 00:42:42.91 & $41: 15: 19.8$ & 22.6 & FJ 73; MMD 1303 \\
\hline M31 B127-24 & $00: 42: 43.02$ & $41: 15: 31.8$ & 22.2 & FJ 19; MMD 1306 \\
\hline M31 B127-25 & $00: 42: 43.03$ & $41: 15: 36.5$ & 21.6 & FJ 20; MMD 1290 \\
\hline M31 B127-26 & $00: 42: 43.07$ & 41:14:08.8 & 22.5 & FJ 139; MMD 1252 \\
\hline M31 B127-27 & $00: 42: 43.27$ & $41: 15: 56.1$ & 21.8 & FJ 7; MMD 1323 \\
\hline M31 B127-28 & $00: 42: 43.79$ & 41:16:01.0 & 21.5 & FJ 8; MMD 1324 \\
\hline M31 B127-29 & 00:42:43.81 & $41: 15: 38.9$ & 22.7 & FJ 319 \\
\hline M31 B127-30 & $00: 42: 44.03$ & $41: 15: 01.7$ & 23.5 & M31 nova 2006-11b \\
\hline M31 B127-31 & 00:42:44.39 & $41: 15: 04.9$ & 22.5 & MMD 1349 \\
\hline M31 B127-32 & 00:42:44.90 & $41: 15: 20.6$ & 21.8 & FJ 74; MMD 1347 \\
\hline M31 B127-33 & 00:42:44.99 & 41:16:03.9 & 22.3 & FJ 324 \\
\hline M31 B127-34 & $00: 42: 45.16$ & $41: 15: 23.2$ & 21.1 & FJ 21; MMD 1266 \\
\hline M31 B127-35 & $00: 42: 45.21$ & 41:16:05.4 & 21.4 & FJ 9 \\
\hline
\end{tabular}


Table 3-Continued

\begin{tabular}{|c|c|c|c|c|}
\hline Designation & $\mathrm{RA}(\mathrm{J} 2000)$ & Dec. (J2000) & $m_{5007}$ & Cross reference ${ }^{\mathrm{a}}$ \\
\hline M31 B127-36 & $00: 42: 45.25$ & $41: 15: 29.2$ & 22.3 & FJ 323; MMD 1295 \\
\hline M31 B127-37 & $00: 42: 45.76$ & 41:16:01.2 & 23.5 & FJ $454 ?$ \\
\hline M31 B127-38 & $00: 42: 45.85$ & 41:16:01.3 & 23.3 & \\
\hline M31 B127-39 & 00:42:46.05 & 41:15:16.1 & 21.6 & FJ 59; MMD 1287 \\
\hline M31 B127-40 & $00: 42: 46.28$ & $41: 15: 22.5$ & 22.3 & FJ 22; MMD 1296 \\
\hline M31 B127-41 & $00: 42: 46.36$ & $41: 15: 46.0$ & 21.5 & FJ 72; MMD 1299 \\
\hline M31 B127-42 & 00:42:46.69 & 41:16:09.6 & 21.2 & FJ 10; MMD 1309 \\
\hline M31 B179-1 & $00: 43: 32.62$ & $41: 17: 20.9$ & 23.2 & AMB 272; MMD 1391 \\
\hline M31 B179-2 & 00:43:32.71 & $41: 18: 32.8$ & 21.4 & MLA 796; AMB 2249; MMD 2767 \\
\hline M31 B193-1 & $00: 43: 40.11$ & 41:36:01.0 & 23.1 & AMB 2331; MMD 647 \\
\hline M31 B472-1 & $00: 43: 41.55$ & $41: 28: 02.1$ & 22.4 & FJ 274; MLA 836; MMD 3022 \\
\hline M31 B472-2 & $00: 43: 43.26$ & $41: 27: 30.2$ & 22.8 & FJ 285; MMD 2706 \\
\hline M31 B193-2 & $00: 43: 49.91$ & $41: 37: 34.3$ & 24.0 & \\
\hline M31 B225-1 & $00: 44: 27.52$ & $41: 22: 29.0$ & 22.6 & MLA 998; AMB 2930; MMD 979 \\
\hline M31 B225-2 & 00:44:31.02 & $41: 22: 25.7$ & 22.9 & WB 445; AMB 334; MMD 978 \\
\hline M31 B386-1 & $00: 46: 30.45$ & 42:01:09.6 & 25.3 & \\
\hline
\end{tabular}

${ }^{a}$ Cross-reference codes: $\mathrm{AMB}=$ Azimlu et al. 2011 (H II regions in M31); FJ = Ford \& Jacoby 1978 (PNe in M31) FJJ = Ford, Jacoby, \& Jenner 1977(PNe in NGC 147 \& NGC 185); HKPN = Hurley-Keller et al. 2004 (PNe in M31); MMD = Merrett et al. 2006 (PNe in M31); MLA = Meyssonnier et al. 1993 (emission-line objects in M31); WB = Walterbos \& Braun 
1992 (emission-line objects in M31). 
Table 4. M31 Globular Clusters in Archival HST [O III] F502N Frames ${ }^{\mathrm{a}}$

\begin{tabular}{llll}
\hline \hline & & & \\
Galaxy \& Cluster & $M_{V}{ }^{\mathrm{b}}$ & ACS Date & WFC3 Date \\
\hline M31-B080 & -7.1 & $2010-12-21$ & \\
M31-B084 & -6.4 & $2010-12-21$ & \\
M31-B096 & -8.0 & $2010-12-21$ & \\
M31-B124 & -9.8 & $2010-12-23$ \\
M31-B127 & -10.1 & $2010-12-23$ \\
M31-B132 & -6.8 & $2010-12-23$ \\
M31-B264 & -7.0 & $2010-12-23$ \\
M31-B477 & -6.1 & $2003-09-27$ & \\
M31-NB21 & -6.7 & & $2010-12-23$ \\
M31-NB39 & -6.6 & & $2010-12-23$ \\
M31-NB41 & -6.5 & $2010-12-23$ \\
M31-NB89 & -6.6 & $2010-12-23$ \\
\hline
\end{tabular}

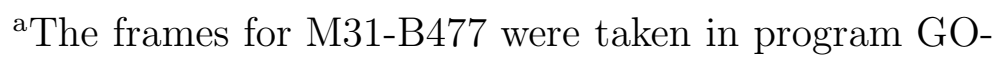
9794 (PI P. Massey). All other frames were taken in GO12174 (PI Z. Li).

${ }^{\mathrm{b}}$ Absolute integrated visual magnitude, calculated from distance and reddening of M31 and the integrated apparent $V$ magnitude 
Table 5. Candidate Planetary Nebula in M31 Cluster B477

\begin{tabular}{lllll}
\hline \hline & & & & \\
Designation & RA (J2000) & Dec. (J2000) & $r^{\mathrm{a}}$ & $m_{5007}$ \\
\hline & & & & \\
M31 B477-1 & $00: 45: 08.41$ & $41: 39: 38.4$ & $1^{\prime \prime} .2$ & 22.3 \\
\hline
\end{tabular}

${ }^{a}$ Angular distance from cluster center

Table 6. Old Globular Clusters in M31 with Nearby PN Candidates

\begin{tabular}{lccc}
\hline \hline & & & \\
GC/PN Designation & & & \\
& & & \\
& & & \\
B075 (J2000) & Dec. (J2000) & Separation (") \\
759 & $00: 42: 08.824$ & $41: 20: 21.25$ & 3.3 \\
B105 & $00: 42: 09.100$ & $41: 20: 22.80$ & \\
534 & $00: 42: 30.747$ & $41: 30: 27.34$ & \\
BH16 & $00: 42: 30.700$ & $41: 30: 24.60$ & 2.8 \\
1360 & $00: 42: 46.090$ & $41: 17: 35.99$ & \\
SK052A & $00: 42: 45.900$ & $41: 17: 36.50$ & 2.3 \\
2598 & $00: 42: 37.315$ & $41: 50: 53.58$ & \\
SK104A & $00: 42: 37.500$ & $41: 50: 56.00$ & 3.0 \\
139 & $00: 45: 44.311$ & $41: 57: 27.80$ & \\
\hline
\end{tabular}

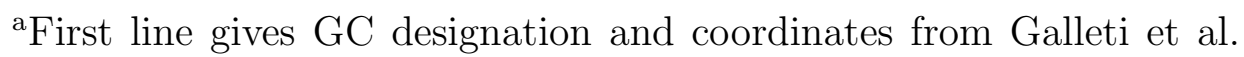
(2004); second line gives PN designation and coordinates from Merrett et al. (2006). 